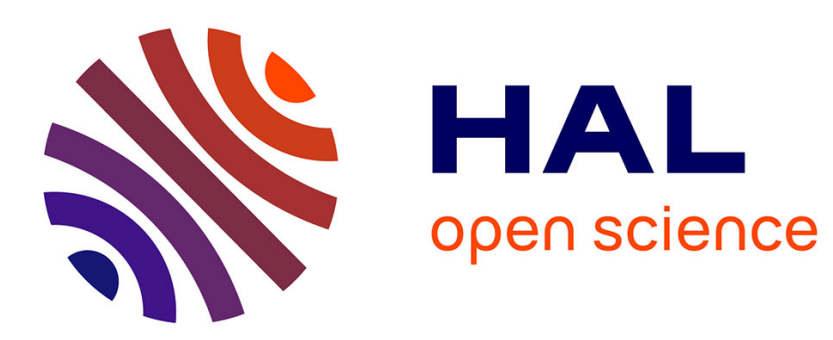

\title{
Chaotic oscillations of the optical phase for multigigahertz-bandwidth secure communications
}

E. Genin, L. Larger, Jean-Pierre Goedgebuer, Min Won Lee, R. Ferriere, X.

Bavard

\section{- To cite this version:}

E. Genin, L. Larger, Jean-Pierre Goedgebuer, Min Won Lee, R. Ferriere, et al.. Chaotic oscillations of the optical phase for multigigahertz-bandwidth secure communications. IEEE Journal of Quantum Electronics, 2004, 40 (3), pp.294-298. 10.1109/JQE.2003.823026 . hal-00096884

\section{HAL Id: hal-00096884 \\ https://hal.science/hal-00096884}

Submitted on 15 Apr 2021

HAL is a multi-disciplinary open access archive for the deposit and dissemination of scientific research documents, whether they are published or not. The documents may come from teaching and research institutions in France or abroad, or from public or private research centers.
L'archive ouverte pluridisciplinaire HAL, est destinée au dépôt et à la diffusion de documents scientifiques de niveau recherche, publiés ou non, émanant des établissements d'enseignement et de recherche français ou étrangers, des laboratoires publics ou privés.

\section{(c)(1)}

Distributed under a Creative Commons Attribution| 4.0 International License 


\title{
Chaotic Oscillations of the Optical Phase for Multigigahertz-Bandwidth Secure Communications
}

\author{
Éric Genin, Laurent Larger, Jean-Pierre Goedgebuer, Min Won Lee, Richard Ferriére, and Xavier Bavard
}

\begin{abstract}
This paper reports a novel optoelectronic nonlinear oscillator, which produces a chaotically phase-modulated light beam over a bandwidth greater than $5 \mathrm{GHz}$. The setup is intended for high-speed chaos-based secure communications. The nonlinear process is performed by the transient response of a fiber interferometer. The flexibility in the definition of such an all-optical nonlinearity features a potentially high-dimensional key space of the encryption process. Modeling, simulations, and experimental results of the dynamical behaviors are reported.
\end{abstract}

Index Terms-Chaos, electrooptic modulation, optical fiber communication, optical resonators, phase modulation.

\section{INTRODUCTION}

D UE TO THE exponential rise of network traffic, and due to the increasing need of privacy in various information exchange situations (such as banking, medicine, and e-business), the study of alternate hardware encryption schemes is currently meeting a growing interest. Whereas quantum cryptography [1] is proposed as a means for providing absolute security in secret key distribution, chaos-based encryption [2]-[7] processes are devoted to the improvement of encryption speed through the use of ultrafast [8], [9] and real-time hardware encoding/decoding systems [10]; conventional algorithm-based methods require time-consuming calculations, thus currently limiting the encryption speed to a few hundred megabits per second. The aim of this paper is to propose a new kind of chaotic oscillator providing a multigigahertz bandwidth and key-flexible structure for the generation of an optical carrier used in chaotic information masking. The experimental setup is first described leading to an unusual two-dimensional (2-D) map-coupled nonlinear delayed band-pass dynamic. Temporal evolutions obtained from the dynamic numerical integration are then reported and compared to the observed experimental behaviors.

\section{EXPERIMENTAL SETUP}

The setup is inspired from a general architecture already described in a previous work dealing with a wavelength chaos

E. Genin, J.-P. Goedgebuer, M. W. Lee, R. Ferriére, and X. Bavard are with Laboratoire d/Optique P.M. Duffieux, University of Franche-Comté, $25030 \mathrm{Be}-$ sançon Cedex, France.

L. Larger is with Laboratoire d/Optique P.M. Duffieux, University of Franche-Comté, 25030 Besançon Cedex, France and also with GTL-CNRS Telecom, 57070 Metz Cedex, France (e-mail: laurent.larger@univ-fcomte.fr).

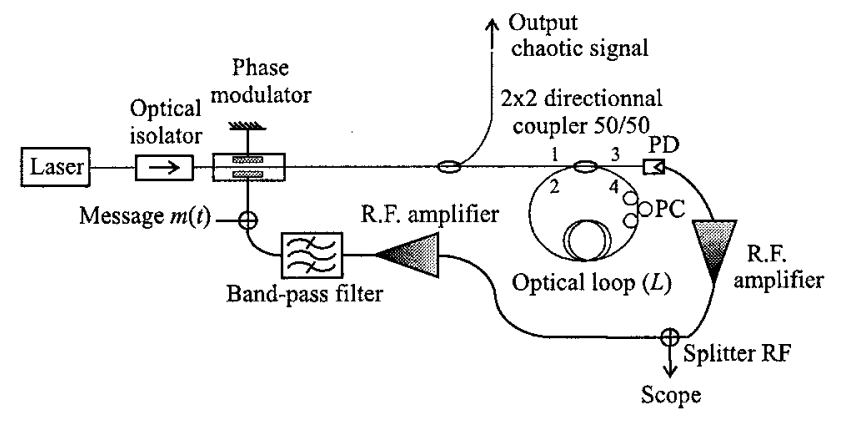

Fig. 1. Experimental setup performing a chaotic optical phase generator with optoelectronic feedback.

generator [4]. This typical architecture was initiated in optics by the pioneering work of Ikeda on nonlinear delayed dynamical systems [11]. The oscillator is organized in an optoelectronic feedback loop, consisting of six characteristic elements: a linear electrical-to-optical converter, an optical-to-optical nonlinear transformation, a delay line, an optical-to-electrical converter, amplifiers, and bandwidth-limiting electronic components. All of these elements can be identified in the setup depicted in Fig. 1.

- The linear electrical-to-optical element is performed by a high-speed integrated electrooptic $\mathrm{LiNbO}_{3}$ phase modulator. While illuminated by a laser source, it generates a phase-modulated output light beam, proportionally to the input electrooptic voltage. The Photline phase modulator of concern has a half-wave tuning rate of $V_{\pi}=5 \mathrm{~V}$ and a modulation bandwidth of $12.5 \mathrm{GHz}$.

- The optical-to-optical nonlinear element is a fiber-ring resonator performed by a $2 \times 250 / 50$ fiber coupler [12], for which port 1 is the input, port 3 the output, and ports 4 and 2 are spliced together. The energy coupling coefficient is $\kappa=1 / 2$, and the ring attenuation is $\gamma=e^{-\alpha L}$. The optical output intensity is thus nonlinearly related to the input optical phase through an interference process, providing that the phase modulation is faster than the cavity round trip. The cavity length is about $1.25 \mathrm{~m}$, resulting in a round-trip time of $T=6.25 \mathrm{~ns}$ and a free spectral range (FSR) of $160 \mathrm{MHz}$, which is hence much smaller than the phase modulator bandwidth. However, due to the small FSR, an ultrastable laser source is required [13] to ensure that the output intensity variations are only caused by the phase modulation and not by some uncontrolled random wavelength drift or fluctuations [14]. The light source used is a Koheras DFB erbium-doped fiber laser 
at $1549 \mathrm{~nm}$, the wavelength of which is slightly tunable through the temperature and which has, at fixed temperature, a wavelength drift of less than $1 \mathrm{MHz}$ and a linewidth smaller than $100 \mathrm{kHz}$. The fiber-ring resonator is mechanically isolated from any external vibration. A polarization controller (PC) is also required inside the fiber loop, so that the interference between the input beam and the one fed back through the fiber ring occurs with the same polarization state. The fiber-ring resonator operates actually as a multiple waves interferometer working in a nonstationary state, since the phase is modulated faster than the cavity round trip. Considering the whole feedback loop setup, the long cavity is also responsible for a delay involved in the oscillator dynamical process. The maximum transmission output power of the ring cavity is approximately $p_{0} \simeq 160 \mu \mathrm{W}$, with a laser power of $1 \mathrm{~mW}$, due to the different losses (isolator, modulator, couplers). One should also notice that any other interferometric device involving a large delay can be used instead of the fiber-ring resonator. The chaotic phase behavior can thus be changed simply by replacing the nonlinear optical-to-optical converter, the latter consisting of any kind of large delay interferometer.

- A detector then converts the former optical output intensity into an electrical signal. This Miteq detector integrates a pigtailed photodiode with a wide-band transimpedance amplifier; covering the $30-\mathrm{kHz}-15-\mathrm{GHz}$ bandwidth, it has a detection sensitivity of $1.98 \mathrm{~V} / \mathrm{mW}$.

- Amplifiers and drivers from $S H F$ with a total gain of $30 \mathrm{~dB}$ over the bandwidth $30 \mathrm{kHz}-25 \mathrm{GHz}$ are finally used in the electronic feedback, in order to ensure an electrooptic voltage swing at least larger than $V_{\pi}$. The dynamical limitation results from the bandwidth combination of the different electronic and optoelectronic components. For chaotic encryption purposes, an RF power combiner can be inserted before the phase modulator feedback, thus allowing an information masking operation through the addition of a small amplitude message into the high-amplitude chaotic feedback signal.

According to the previous description of the individual elements in the experimental setup, a simple differential model will be addressed in the following section.

\section{THEORY AND MODELING}

On the basis of the different elements constituting the feedback loop, the dynamical process can be derived from the RF filtering applied to the delayed interference function performed by the fiber-ring cavity. It is here assumed that the overall dynamics is only determined by the slowest components in the feedback loop, i.e., the electronic part of the feedback. The time fluctuations $\phi(t)$ are then supposed to be ruled by the linear electronic feedback filtering - impulse response $h(t)$ - which is applied to the optical intensity fluctuations detected at the input of the electronic feedback ( $p_{3}$, the optical input of the photodiode): $\phi(t)=\left[h \star p_{3}\right](t)$. Since $h(t)$ is not known and might be quite complicated according to the cascaded influence of the different electronic parts, we assume that the actual dynamic can be ap- proximated by the simplest filtering of the same nature i.e., a second-order bandpass filter with the same low and high cutoff frequencies. The dynamics of $\phi(t)$ in the Laplace domain would then be

$$
\Phi(p)=H(p) \cdot P_{3}(p)=\frac{G \tau_{1} p}{\left(1+\tau_{1} p\right)\left(1+\tau_{2} p\right)} \cdot P_{3}(p)
$$

where $\Phi(p)=\operatorname{TL}[\phi(t)]$ is the Laplace transform of the optical phase modulation $\phi(t)$ induced electrooptically, $P_{3}(p)$ is the Laplace transform of the optical power modulation $p_{3}(t)$ detected by the photodiode, and $H(p)$ is representative in the Laplace domain of the filtering performed by the electronic feedback loop. $f_{c 1}=1 /\left(2 \pi \tau_{1}\right)$ (approximately $30 \mathrm{kHz}$ ) and $f_{c 2}=1 /\left(2 \pi \tau_{2}\right)$ (approximately $12 \mathrm{GHz}$ ) are respectively the low and high cutoff frequencies of the feedback-loop bandwidth (associated with the response times $\tau_{1}$ and $\tau_{2}$ ). $G=\pi \cdot K \cdot S / V_{\pi}$ is the total optoelectronic gain of the feedback loop, comprising the RF amplifier gain $K$, the electrooptic tuning rate $V_{\pi}$, and the photodiode sensitivity $S$. The inverse Laplace transform then allows us to derive from (1) the differential law ruling the time fluctuations of the modulated optical phase $\phi(t)$. A second-order scalar delay differential equation is obtained, involving on the right-hand side the derivative of the optical power detected by the photodiode

$$
\phi(t)+\left(\tau_{1}+\tau_{2}\right) \frac{\mathrm{d} \phi}{\mathrm{d} t}(t)+\tau_{1} \tau_{2} \frac{\mathrm{d}^{2} \phi}{\mathrm{d} t^{2}}(t)=G \tau_{1} \frac{\mathrm{d} p_{3}}{\mathrm{~d} t}(t) .
$$

The right-hand-side term acts as a driving force strongly decorrelated from the instantaneous variations, due to the delay and the nonlinear transformation performed by the fiber-ring cavity on the phase-modulated light beam. The optical power $p_{3}(t)$ detected by the photodiode is derived from the equations of a $2 \times 2$ fiber coupler with a coupling coefficient $\kappa$ and coupling losses $\rho$ as follows:

$$
\begin{aligned}
& E_{3}=\sqrt{\rho}\left(\sqrt{\kappa} E_{1}+i \sqrt{1-\kappa} E_{2}\right) \\
& E_{4}=\sqrt{\rho}\left(i \sqrt{1-\kappa} E_{1}+\sqrt{\kappa} E_{2}\right)
\end{aligned}
$$

where $E_{1}(t)=\sqrt{p_{0}} e^{i \phi(t)}$ is the phase-modulated light beam with constant intensity, $E_{3}(t)=\sqrt{p_{3}(t)} e^{i \varphi_{3}(t)}$ is the output field, and $E_{2}(t)=\sqrt{\gamma} E_{4}(t-T) e^{i \phi_{L}}=\sqrt{\gamma} E_{4 T} e^{i \phi_{L}}$ is the delayed optical feedback in the fiber-ring resonator; $\phi_{L}$ is the phase shift due to the propagation through the ring at a fixed laser wavelength $\lambda\left(\phi_{L}=2 \pi n L / \lambda\right), \gamma$ corresponds to the fiber and coupler losses, and $E_{4_{T}}$ is the optical field at port 4 delayed in time, $E_{4_{T}}=\sqrt{p_{4_{T}}} e^{i \varphi_{4_{T}}}$. The instantaneous effect of the fiber-ring resonator consists of a two-waves interference between an input field $E_{1}$ and the feedback field $E_{2}$. The output power $p_{3}$ detected by the photodiode can thus be expressed as

$$
\begin{aligned}
p_{3}(t)=\rho p_{0} & {\left[\kappa+(1-\kappa) \gamma \frac{p_{4_{T}}}{p_{0}}\right.} \\
& \left.-2 \sqrt{\gamma \kappa(1-\kappa) \frac{p_{4_{T}}}{p_{0}}} \sin \left(\varphi_{4_{T}}+\phi_{L}-\phi(t)\right)\right]
\end{aligned}
$$

where $p_{4}$ and $\varphi_{4}$ are determined by a 2-D mapping due to the successive path of the field $E_{4}$ through the fiber ring and are given by (5), shown at the bottom of the following page. 


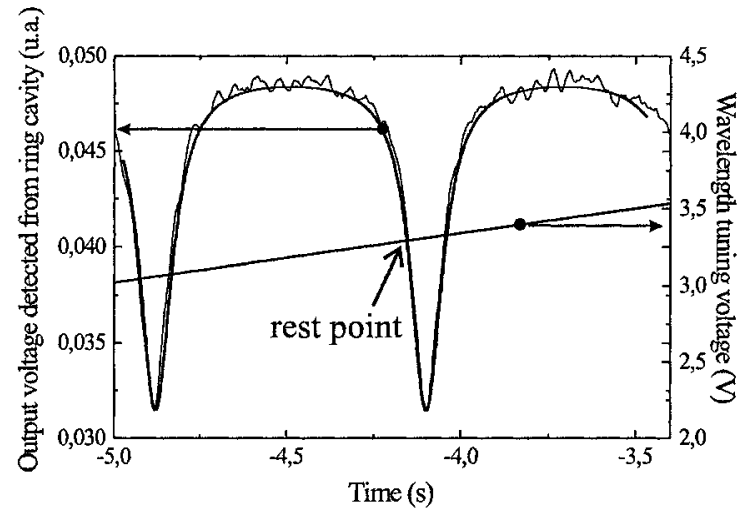

Fig. 2. Static optical filter performed by the fiber-ring cavity, while the laser wavelength is slowly scanned in time using temperature control.

In (5), one could notice the influence of the electrooptic phase modulation $\phi(t)$, since this modulation is performed faster than the ring round-trip time $T$. When no phase modulation is applied $\left(\phi(t)=\phi_{0}\right)$, (4) and (5) are equivalent to a 2-D mapping. The asymptotic behavior of this mapping corresponds to the typical spectral filtering of a fiber-ring cavity, shown as follows:

$$
p_{3}=p_{0} \frac{(\sqrt{\kappa}-\sqrt{\gamma})^{2}+4 \sqrt{\kappa \gamma} \sin ^{2}\left(\phi_{L} / 2\right)}{(1-\sqrt{\kappa \gamma})^{2}+4 \sqrt{\kappa \gamma} \sin ^{2}\left(\phi_{L} / 2\right)}
$$

where

$$
\phi_{L}=\frac{2 \pi n L}{\lambda}
$$

Fig. 2 shows the filter profile observed when the fiber-ring cavity is illuminated by the laser, the wavelength of which is slightly varied using laser temperature control. An experimental curve is superimposed on the theoretical one, verifying the expected interferometric behavior of the fiber-ring cavity and allowing one to measure the finesse of the spectral filter $(F \simeq 8.4$, the value of which is consistent with the estimated experimental losses in the ring cavity $\gamma \simeq 1$ and $\rho \simeq 0.88$ ). The parameter $\phi_{L}$ appearing in (4) and (5) is practically tuned using the laser temperature control and estimated from the experimental curve in Fig. 2. A typical value of $\lambda$ used in the experimental study of the dynamical behavior corresponds to the rest point indicated by the arrow i.e., $\phi_{L}=0.34 \mathrm{rad}$. That particular value is chosen because it corresponds to the highest small-signal gain of the oscillator loop, with which the best conditions for instabilities and chaotic oscillations are obtained.

\section{EXPERIMENTAL AND NUMERICAL DYNAMICAL BEHAVIORS}

Equations (2)-(5) are used to perform numerical integrations, thus giving numerous and various simulated dynamical regimes
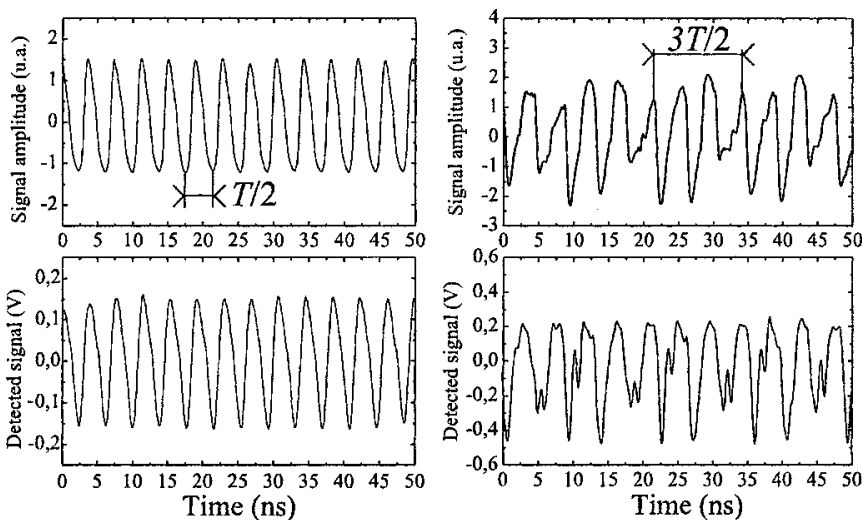

Fig. 3. Periodic waveforms obtained for low values of the feedback loop gain. Top: simulated with $\beta=2.5$ (left) and $\beta=2.8$ (right). Bottom: experimental with $\beta=2.5$ (left) and $\beta=2.8$.

that should be observed experimentally. The integration procedure is based on a fourth-order Runge-Kutta algorithm. The obtained regimes depend on the different possible parameter values (such as wavelength, intensity, or optoelectronic feedback gain). A typical bifurcation parameter is the total feedback loop gain $\beta=G p_{0} / 2$ of the nonlinear delayed oscillator. This unit free gain can actually be varied experimentally in the range $[0.1,5.5]$ through the laser intensity or, as actually done, using a tuning gain voltage available in RF amplifiers, thus allowing a qualitative comparison between experiment and modeling. The other parameters are fixed to the values previously defined ( $p_{0} \simeq 160 \mu \mathrm{W}, \phi_{L} \simeq 0.34 \mathrm{rad}$ ) and measured experimentally.

For low values of the bifurcation parameter, the feedback loop gain is not high enough to initiate oscillations, and a zerostable steady state is observed. When the gain is increased, a periodic oscillation is first observed both numerically and experimentally from approximately $\beta=1.9$ to $\beta=2.65$, as shown by the two left traces in Fig. 3. The frequency of about $320 \mathrm{MHz}$ is consistent with the second harmonic of the characteristic round-trip frequency $1 / T$, as usually observed in delay dynamics. From $\beta=2.65$, more complex nearly periodic behaviors are observed in experiments (lower right trace in Fig. 3) as well as in simulations (upper right trace in Fig. 3). They exhibit a fundamental frequency of about $100 \mathrm{MHz}$, which corresponds to $1 / 3$ of the previous periodic oscillation; such subharmonic regimes are also characteristic in the bifurcation scenario of delay dynamics.

When further increasing $\beta$, the oscillator enters chaotic regimes, and typical ones are reported with their spectrum in Fig. 4. The time traces appear as a high-amplitude noise signal, and their spectrum extends with a nearly constant amplitude

$$
\begin{aligned}
& p_{4}(t)=\rho p_{0}\left[1-\kappa+\gamma \kappa \frac{p_{4_{T}}}{p_{0}}+2 \sqrt{\gamma \kappa(1-\kappa) \frac{p_{4_{T}}}{p_{0}}} \sin \left(\phi_{L}-\phi(t)+\varphi_{4_{T}}\right)\right] \\
& \varphi_{4}(t)=\phi(t)+\frac{\pi}{2}-\arctan \left[\frac{\sqrt{\kappa \gamma p_{4_{T}}} \cos \left(\phi_{L}-\phi(t)+\varphi_{4_{T}}\right)}{\sqrt{(1-\kappa) p_{0}}+\sqrt{\kappa \gamma p_{4_{T}}} \sin \left(\phi_{L}-\phi(t)+\varphi_{4_{T}}\right)}\right]
\end{aligned}
$$



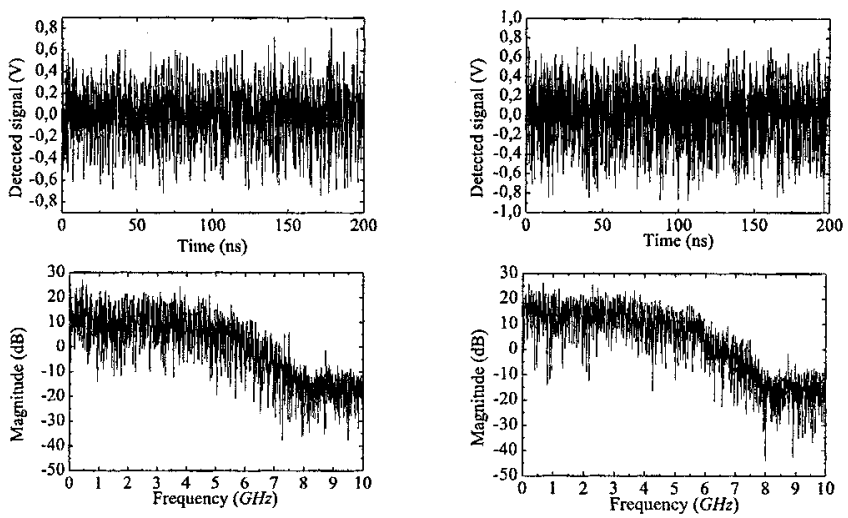

Fig. 4. Chaotic traces (upper) and their spectra (lower) for $\beta=4.0$ (left) and $\beta=5.0$ (right).

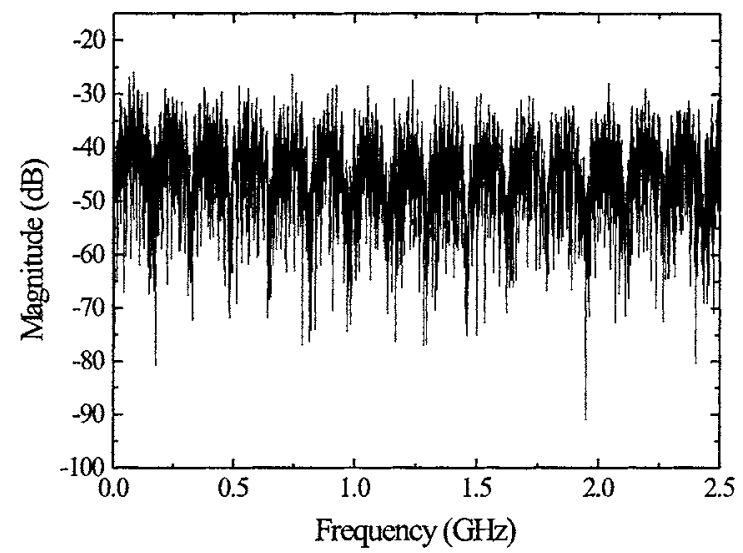

Fig. 5. Low-frequency spectrum in the chaotic regime: white noise-like spectrum with antiresonant dips at frequencies corresponding to the fiber-ring FSR.

level over a multigigahertz bandwidth related to the one of the optoelectronic oscillator feedback loop (from a few tens of kilohertz to approximately $5 \mathrm{GHz}$ ). It should be noticed that the chaotic spectrum is more and more extended when further increasing the bifurcation parameter. A fine resolution of the RF spectra (Fig. 5) reveals typical sharp frequency rejection filtering performed by the optical ring cavity: each $\mathrm{RF}$ frequency of the phase modulation that is a multiple of the cavity FSR (160 MHz) verifies an antiresonant feedback condition in the optical cavity.

A more global overview of the numerous dynamical regimes observed for a fixed $\phi_{L}$ and an increasing feedback gain $\beta$ are given by the experimental and numerical bifurcation diagrams in Fig. 6. The horizontal axis corresponds to the bifurcation parameter (the RF amplification gain), which was slowly increased in time, and the vertical axis represents in gray scale the probability density of the dynamical variable (the voltage applied to the phase modulator) for each regime corresponding to a given value of $\beta$. Although the dynamical model was established with a rough assumption (differential process corresponding to the linear filtering effect of a second-order bandpass filter), a good qualitative agreement can be noticed, in terms of the global bifurcation diagram shape, but also in terms of the bifurcation positions along the horizontal axis.
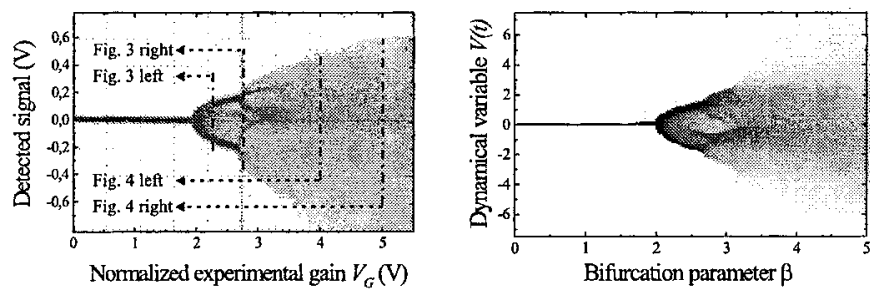

Fig. 6. Bifurcation diagrams with respect to the feedback loop gain $\beta$ (left, experimental; right, numerical).

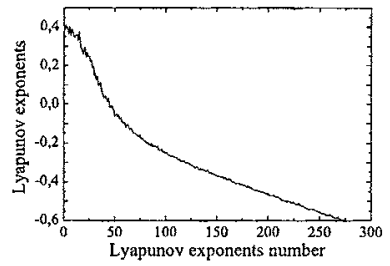

(a)

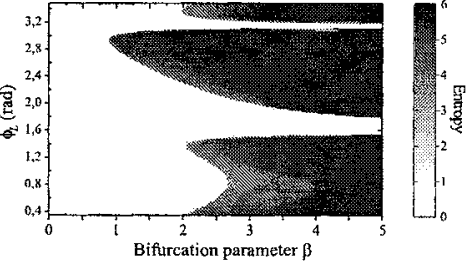

(b)
Fig. 7. Quantitative measures of the dynamical regimes. (a) Lyapunov spectrum for $\beta=5.0$ and $\phi_{L}=0.34$. (b) Statistical entropy in the $\left(\beta, \phi_{L}\right)$ plane.

Quantitative measures of the dynamical regimes have been explored numerically from the model. The results are reported in Fig. 7. Fig. 7(a) represents a Lyapunov spectrum for a given chaotic regime and shows that there exist many positive Lyapunov exponents (more than 40). This spectrum allows us to calculate the Lyapunov dimension, which is found to be around 108 , thus indicating a high complexity of the corresponding chaotic attractor. Fig. 7(b) corresponds to the statistical entropy of many different dynamical regimes with respect to two parameters, $\beta$ and $\phi_{L}$. The chaotic regimes are obtained for maximum entropy corresponding to dark regions, whereas steady states or periodic and quasi-periodic regimes are characterized by the white or gray regions. This diagram allows us to define more precisely parameter space regions for which chaos can be obtained.

\section{CONCLUSION}

We reported a new nonlinear delay optoelectronic oscillator operating with the dynamical variable optical phase. Important characteristics of this oscillator are its wide bandwidth starting from a few tens of kilohertz up to more than $5 \mathrm{GHz}$, its bandpass dynamical process, and the realization of an all-optical 2-D map transformation providing simultaneously a time delay and a nonlinear process for the dynamic. The delay nonlinear transformation is a fiber-ring cavity operating as a very long optical path difference (OPD) interferometric device; it is carried out using standard fiber-based components. The time delay involved in the dynamics is much larger than the response time of the electronic feedback, thus allowing high-complexity dynamical behaviors which is typical of time-delay systems. A simple dynamical model was derived from the bandpass nature of the electronic feedback loop and from the nonstationary interference function of the fiber ring cavity. Good qualitative and quantitative agreement was found between the dynamical regimes obtained numerically and the ones observed experimentally. The established model also allowed us to estimate numer- 
ically some quantitative measure of various dynamical regimes, such as the entropy and the Lyapunov spectrum.

The flexibility in the realization of the nonlinear delay device in this oscillator might be of great interest in the context of encryption using chaotic carriers. The setup is able to produce a fast chaotic carrier that could be used for multi-Gb/s data encryption. Moreover, its realization flexibility through the use of any kind of long OPD interference effect would allow the definition of a large key-space of the encryption process. In terms of dynamical complexity, we showed through numerical simulations that the Lyapunov dimensions are comparable with the ones typically obtained on scalar delay differential systems involving scalar nonlinear process $\left(D_{L} \simeq 100\right)$. Further studies are nevertheless required to investigate more precisely the complexity of this new kind of delay dynamics, with respect to different parameters such as the delay.

\section{REFERENCES}

[1] N. Gisin, "Quantum cryptography," Rev. Mod. Phys., vol. 74, pp. $145-195,2002$.

[2] L. M. Pecora and T. L. Carroll, "Synchronization in chaotic systems," Phys. Rev. Lett., vol. 64, pp. 821-824, 1990.

[3] C. Mirasso, P. Colet, and P. Garcia-Fernandez, "Synchronization of chaotic semiconductor lasers: Application to encoded communications," IEEE Photon. Technol. Lett., vol. 8, pp. 209-211, 1996.

[4] L. Larger, J.-P. Goedgebuer, and F. Delorme, "Optical encryption system using hyperchaos generated by an optoelectronic wavelength oscillator," Phys. Rev. E, vol. 57, pp. 6618-6624, 1998.

[5] G. Van Wiggeren and R. Roy, "Optical communication with chaotic waveforms," Phys. Rev. Lett, vol. 91, pp. 3547-2550, 1998.

[6] V. Annovazzi-Lodi, S. Donati, and A. Scire, "Synchronization of chaotic injected-laser systems and its applications to optical cryptography," IEEE J. Quantum Electron., vol. 32, pp. 953-959, June, 1996.

[7] S. Donati and C. Mirasso, "Optical chaos and its applications to crptography," IEEE J. Quantum Electron., vol. 38, pp. 1138-1196, Sept. 2002. Special Issue.

[8] I. Fischer, Y. Liu, and P. Davis, "Synchronization of chaotic semiconductor laser dynamics on subnanosecond time scales and its potential for chaos communication," Phys. Rev. A, vol. 62, pp. 1-4, 2000.

[9] S. Tang and J. M. Liu, "Chaotic pulsing and quasiperiodic route to chaos in semiconductor laser with delayed opto-electronic feedback," IEEE J. Quantum Electron., vol. 37, pp. 329-336, Mar. 2001.

[10] S. Sivaprakasam and K. A. Shore, "Message encoding and decoding using chaotic external-cavity diode lasers," IEEE J. Quantum Electron., vol. 36, pp. 35-39, Jan. 2000.

[11] K. Ikeda and H. Daido, "Optical turbulence: Chaotic behavior of transmitted light from a ring cavity," Phys. Rev. Lett., vol. 45, pp. 709-712, 1980.

[12] L. F. Stokes, M. Chodorow, and H. J. Shaw, "All-single-mode fibre resonators," Opt. Lett., vol. 7, pp. 288-290, 1982.

[13] Y. Imai and T. Tamura, "Coherence effect on nonlinear dynamics in fiber-optic ring resonator," Opt. Commun., vol. 195, pp. 259-265, 2001.

[14] Y. Ohtsuka, "Analysis of a fiber-optic passive loop-resonator gyroscope: Dependence on resonator parameters and light-source coherence," $J$. Lightwave Technol., vol. LT-3, pp. 378-384, Apr. 1985. 